J. Clin. Chem. Clin. Biochem.

Vol. 25, 1987, pp. 91-94

(C) 1987 Walter de Gruyter \& Co.

Berlin - New York

\title{
Chemical Detection of Leukocytes in Urine by Means of a New Multiple Test Strip
}

\author{
By D. Kutter, Gracia Figueiredo and Laure Klemmer
}

Pharmaceutical Institute (Director: Prof. Dr. pharm. U. Kesselring) University of Lausanne

(Received May 3/September 12, 1986)

Summary: The performance of the leukocyte test area of the new multiple urine test strip, Multistix-10$\mathrm{SG}^{\circledR}$, was evaluated in both field trials and model experiments. The detection limit was found to be adequate for routine purposes. Unspecific reactions are rare and generally indicated by the results of the other test areas.

\section{Introduction}

The well tried multiple test strip, N-Multistix-SG ${ }^{\circledR}$ (Bayer-AMES), has been augmented by an additional test area for the chemical detection of leukocytes. It is now marketed under the name Multistix-10-SG ${ }^{\circledR}$. The new test is based upon the esterase activity of granulocytes. 3-Hydroxy=5-phenyl-pyrrole esterified with an amino acid is used as substrate. Hydrolysis of this ester by the esterase releases 3-hydroxy-5phenyl-pyrrole, which in turn reacts with a suitable diazonium salt, yielding a violet azo-dye (fig. 1).

The aim of this study was to investigate the clinical performance of this test in an extensive clinical trial, with particular emphasis on the detection limit and specificity.
Any reagent used with urine will be subject to a variety of unconstant reaction conditions caused by the highly variable chemical composition of urine. Previous experience with competitive tests has moreover shown that the esterase reaction of urine is increased by lysis of any leukocytes present. Leukocyte lysis is far from constant, depending on other variables such as $\mathrm{pH}$ and osmolarity. For these reasons it is impossible to characterize a reagent by just one detection limit. A statistical approach is generally preferred, by establishing a curve, plotting the percentage of positive results against parameter concentration. The two characteristic points of this curve are $D_{10}$ (low detection limit, representing the concentration detected in $10 \%$ of a certain number

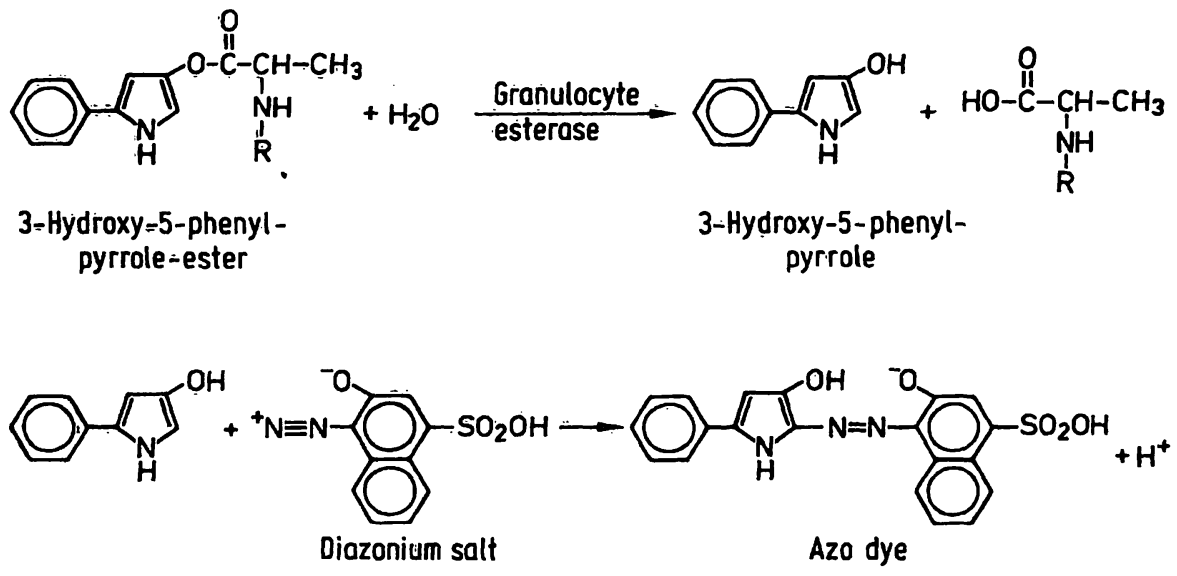

Fig. 1. Reaction mechanism of the leukocyte test area of Multistix 10-SG® (Bayer-AMES). 
of specimens), and $D_{90}$ (practical detection limit, being the concentration that yields a positive test in $90 \%$ of the samples). This curve can be determined either by field trial on a large number of specimens or by model experiments, and it should be located as close as possible to the highest concentration accepted as physiological, without the $D_{10}$-value dropping into the physiological area (1).

The investigation of specificity mainly consists of model experiments to verify unspecific reactions, which may be predictable from the reaction mechanism, stated by the manufacturers or known from similar competitive products. Discrepancies between test and reference method in the field trial may also disclose unspecific reactions.

When studying cellular elements in urine, lysis often accounts for apparently false positive reactions of the test, which also detects free cellular enzymes. High $\mathrm{pH}$ and low specific gravity are commonly known to be responsible for rapid lysis (2-4). It was therefore investigated whether additional consideration of the test areas for $\mathrm{pH}$ and specific gravity could cut down the number of apparent discrepancies between chemistry and microscopy.

Today many laboratories restrict microscopic examination to specimens giving at least one positive reaction for the four parameters blood, leukocytes, protein and nitrite. This procedure has been found acceptable by many authors $(5-9)$. The problem of whether the new leukocyte test would fit into such a "test strip sieve" was studied in a field trial.

\section{Materials and Methods}

Urines used throughout this trial were exclusively fresh morning specimens, generally not cleanly voided. Testing was performed according to the instructions given by the manufacturers. The leukocyte test area was compared with the colour scale after two minutes. Single tests per specimen were performed by one operator, whose colour vision had been found to be excellent by means of a home made testing device (1).

Chamber counting of uncentrifuged urine in a Fuchs-Rosenthal counting chamber according to Stansfeld \& W'ebb (10) was used as a reference method. Counting was performed by one operator unaware of the chemical results. To ensure a margin of error not exceeding $\pm 10 \%$ a minimum of 100 elements were counted.

\section{Results}

A first attempt to characterize the detection limit was made in a large field trial. Four hundred and twenty two urines were tested with the test strip and the result was compared with the number of leukocytes per microlitre determined by the reference method. The results are represented in figure 2 .

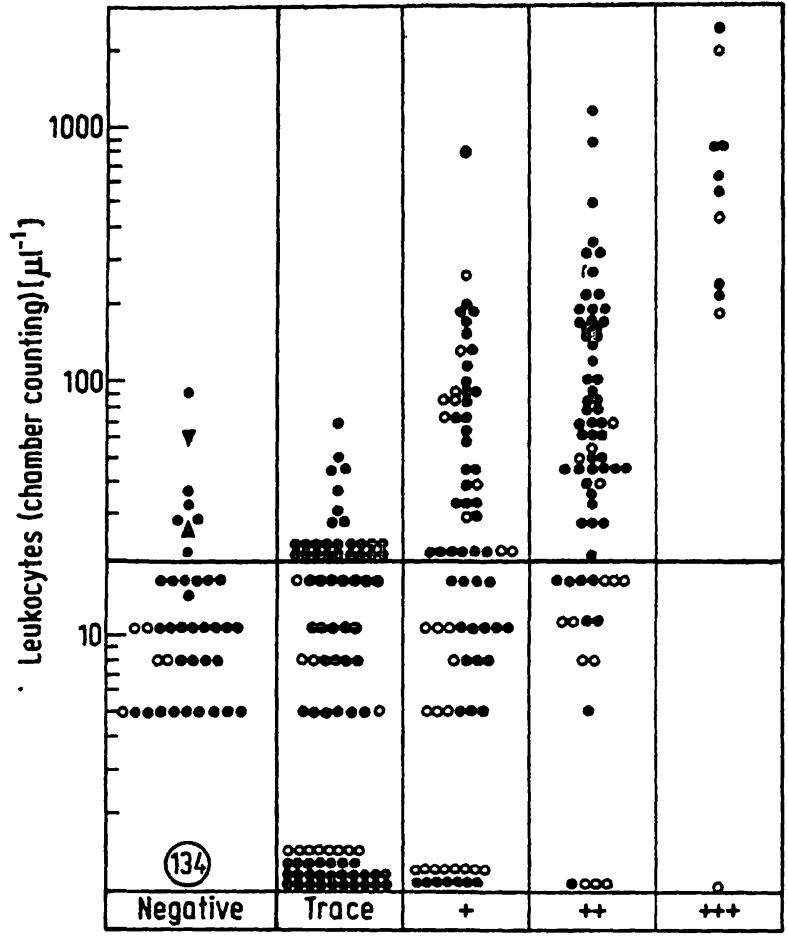

Fig. 2. Results of the leukocyte test area of Multistix-10-SG ${ }^{\circledR}$ (Bayer-AMES) compared with quantitative microscopy. $n=422$. Open circles represent specimens in which values for $\mathrm{pH}$ and/or specific gravity suggest lysis of the leukocytes. $\nabla$ specimen containing $3.0 \mathrm{~g} / \mathrm{l}$ protein. $\Delta$ specimen containing $50 \mathrm{~g} / \mathrm{l}$ glucose.

After classifying the cell counts in ranges we tried to obtain a typical curve by plotting the percentage of positive reactions in the different ranges against leukocyte concentration. The $D_{90}$-point of this curve (practical detection limit) can easily be located at approximately 20 leukocytes per microlitre (fig. 3). A rather high percentage of samples with a positive test and negative microscopy prevents, however, the definition of the $D_{10}$-value. This may be caused either by a high frequency of specimens containing com-

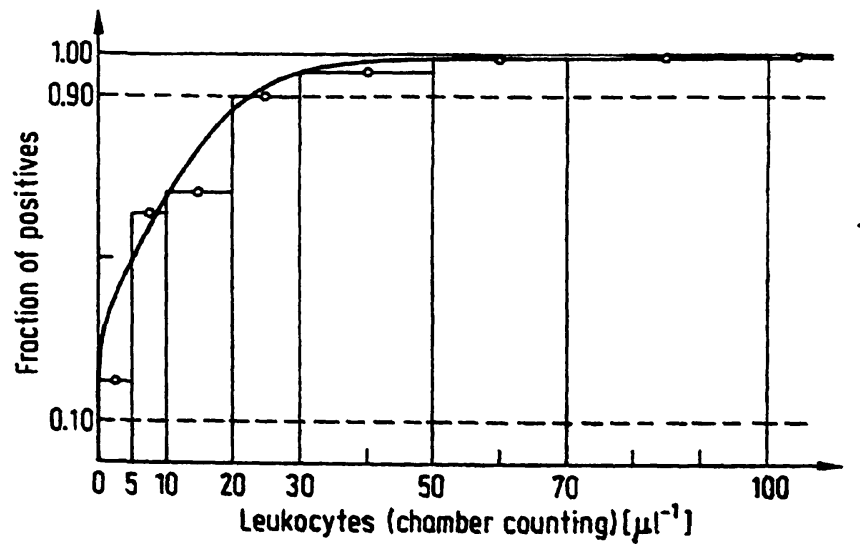

Fig. 3. Approximate detection limit curve determined in a field trial on 422 specimens. Open circles represent the fraction of positives in the different ranges of concentration. 
pletely lysed leukocytes, by true false positive reactions due to cross-reacting metabolites or by oversensitivity of the reagent with an extremely low $D_{10^{-}}$ value.

In order to eliminate the latter possibility the following model experiment was performed: 20 urine specimens yielding a negative test strip reaction and showing no leukocytes or fragments of leukocytes under the microscope were spiked with increasing proportions of a specimen containing 1000 leukocytes per microlitre. From the results we draw the ideal curve shown in figure 4.

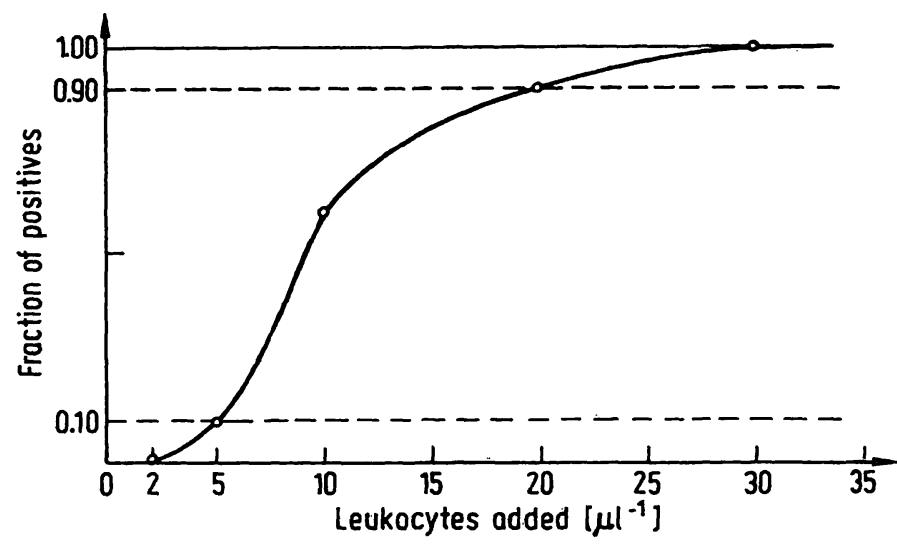

Fig. 4. Detection limit curve obtained by spiking 20 leukocytefree specimens with increasing concentrations of leukocytes from a specimen containing $1000 \mu^{-1}$ leukocytes.

Tab. 1. Results of the leukocyte test area of Multistix-10-SG ${ }^{\circledR}$ (Bayer-AMES) in the presence of glucose.

\begin{tabular}{lll}
\hline $\begin{array}{l}\text { Glucose } \\
(\mathrm{g} / \mathrm{l})\end{array}$ & $\begin{array}{l}\text { Urine } \mathrm{A} \\
\left.\text { (Leukocytes: } 32 \mu \mathrm{l}^{-1}\right)\end{array}$ & $\begin{array}{l}\text { Urine B } \\
\left.\text { (Leukocytes: } 104 \mu \mathrm{l}^{-1}\right)\end{array}$ \\
\hline 0 & + & ++ \\
5 & + & ++ \\
10 & Trace & + \\
20 & Negative & + \\
40 & Negative & Negative \\
\hline
\end{tabular}

Tab. 2. Results of the leukocyte test area of $M$ Multistix-10-SG ${ }^{\circledR}$ (Bayer-AMES) in the presence of protein. Protein was added in form of serum.

\begin{tabular}{lll}
\hline $\begin{array}{l}\text { Protein } \\
\text { added } \\
(\mathrm{g} / \mathrm{l})\end{array}$ & $\begin{array}{l}\text { Urine A } \\
\text { (Leukocytes: } 32 \mu \mathrm{l}^{-1} \text { ) }\end{array}$ & $\begin{array}{l}\text { Urine B } \\
\text { (Leukocytes: } 104 \mu \mathrm{l}^{-1} \text { ) }\end{array}$ \\
\hline 0 & + & ++ \\
0.75 & + & ++ \\
1.50 & + & ++ \\
3.00 & Trace & + \\
5.00 & Negative & + \\
\hline
\end{tabular}

The investigation of specificity was restricted to the following points:

The inhibition by extreme concentrations of glucose stated by the producers is confirmed. Its extent is shown in table 1.

Inhibition by protein is described for both formulations of Cytur-Test (Boehringer Mannheim) $(11,12)$. Table 2 shows that it also applies to this test.

True false positives with saliva or with formaldehyde ( $>400 \mathrm{mg} / \mathrm{l}$ ) described for the competitive products are also encountered with this test. Cat urine always yields a strong false positive reaction. This is not the case with urine from dogs.

In an expanded field trial, Multistix-10-SG ${ }^{\circledR}$ was used for the "test strip sieve", consisting of the parameters blood, leukocytes, protein and nitrite. In 712 cases the results of this sieve were compared with systematic quantitative microscopy. The results are shown in table 3 .

\section{Comments}

If we consider leukocyte concentrations of $20 \mu \mathrm{l}^{-1}$ and more as pathological, we find only 8 true false negatives among the 422 specimens of the first field trial (upper left quadrant in fig. 2). Two of these may

Tab. 3. Efficiency of the test strip sieve using Multistix-10-SG ${ }^{\circledR}$ (Bayer-AMES) compared with urine microscopy $(\mathrm{n}=$ 712). 352 specimens were negative by both microscopic and chemical examination, comprising the groups marked*).

\begin{tabular}{lll}
\hline Microscopic results & \multicolumn{2}{l}{ Test strip sieve } \\
\cline { 2 - 3 } & Positive & Negative \\
\hline
\end{tabular}

$\left.\begin{array}{lll}\hline \text { Leukocytes }\left(\mu \mathrm{l}^{-1}\right) & & \\ 0 & 52 & * \\ 5-10 & 36 & 7 \\ 11-20 & 44 & 6\end{array}\right\}_{\text {nignificant }}$

Erythrocytes $\left(\mu 1^{-1}\right)$

\begin{tabular}{|c|c|c|}
\hline $\begin{array}{l}0 \\
5-10 \\
11-20 \\
21-50 \\
50\end{array}$ & $\left.\begin{array}{l}37 \\
48 \\
70 \\
59 \\
32\end{array}\right\} 209$ & $\left.\begin{array}{l}*) \\
6 \\
5 \\
2\end{array}\right\} 13=5.9 \%$ \\
\hline Bacteria & 58 & 4 \\
\hline $\begin{array}{l}\text { Hyaline casts } \\
\text { Granular casts } \\
\text { Leukocyte casts }\end{array}$ & $\begin{array}{l}2 \\
1 \\
1\end{array}$ & - \\
\hline Crystals & 14 & 4 \\
\hline
\end{tabular}


be explained by high glucose $(\boldsymbol{\nabla})$ or high protein $(\Delta)$. Only 3 out of the remaining 6 samples show specific gravity of $1.030 \mathrm{~kg} / \mathrm{l}$. On the other hand we find extreme specific gravity in about half of the positive samples. We cannot therefore confirm the inhibitory effect of high specific gravity stated by the producer.

Our results clearly show that we cannot afford to consider "trace"-results irrelevant, as the number of true positive specimens in this group is not negligible.

Considering the 8 false negatives and the 128 true positives (upper right quadrant in fig. 2) we calculate an acceptable sensitivity of 0.94 . If we admit that all the 118 results in the lower right quadrant of figure 2 represent false positives, we obtain a rather poor specificity of 0.59 . This group, however, is certain to contain a number of specimens containing lysed leukocytes. If we consider $\mathrm{pH} \geqslant 7$ and/or specific gravity $<1.020 \mathrm{~kg} / \mathrm{l}$ as indicative for lysis, 38 specimens may be subtracted from this group (open circles in fig. 2), with a slight improvement of specificity to 0.68 . Results published by Kierkegaard et al. (2), however, show that lysis is a very common phenomenon not restricted to samples with high $\mathrm{pH}$ and low specific gravity. It is therefore impossible to rule out lysis for the remaining samples. On the other hand, unspecific reactions by unidentified urinary metabolites cannot be entirely excluded, especially for the "trace"-results. Such unidentified substances may be capable of hydrolysing the substrate, or they could be metabolites capable of reacting with the diazonium salt. As the model experiment shown in figure 4 clearly indicates a low detection limit of 5 leukocytes per microlitre, a possible oversensitivity of the reagent may be ruled out. From our experience with a very similar competitive product we conclude that true false positives must be rare (13): we obtained almost identical results in the field trial on 320 unselected

\section{References}

1. Kutter, D. (1985) Clinical Biochemistry Nearer the Patient, Churchill Livingstone, Edinburgh, pp. 139-149.

2. Kierkegaard, H., Feldt-Rasmussen, U., Horder, M., Andersen, H. J. \& Jorgensen, P. J. (1980) Scand. J. Lab. Invest. $40,259-261$.

3. Schönberner, H. (1981) Symposium Vorfelddiagnostik, Gesellschaft für klinische Chemie und Laboratoriumsdiagnostik der DDR, Nahmitz.

4. Triger, D. R. \& Smith, J. W. G. (1966) J. Clin. Pathol. 19, 443-447.

5. Bonard, C., Weber, E., Koller, P. U., Willamowski, K. D. \& Bachmann, F. (1982) Dtsch. Med. Wochenschr. 107, 249251. patient urines. The constellation test strip positive/ microscopy negative was, however, encountered only twice in a group of 78 healthy males. This would mean that in reality most of our "false positives" are true positives, representing specimens in which leukocytes have been lysed. This not only means that the reagent has an acceptable' specificity. It also demonstrates the superiority of chemistry over microscopy, which is incapable of detecting lysed elements.

We do not want to discuss the adequacy of the "test strip sieve", which has been the subject of many publications. We just want to point out that Multistix-10 ${ }^{\circledR}$ may be used for this purpose. In our field trial only $0.8 \%$ of the cases of significant leukocyturia were missed. The test strip is slightly less reliable for red blood corpuscles, owing to the somewhat lower detection limit of the "blood" area.

\section{Conclusion}

It is generally admitted that leukocyte concentrations below $5 \mu \mathrm{l}^{-1}$ have no clinical significance and that concentrations of $20 \mu \mathrm{l}^{-1}$ and more are significant. Values in the intermediate range may be of interest in special cases only. With its low detection limit of $5 \mu \mathrm{l}^{-1}$ and its practical detection limit of $20 \mu \mathrm{l}^{-1}$ we consider this reagent acceptable for clinical purposes. The performance of the reagent is improved when it is used in the test strip sieve, where possible inhibitions by glucose or protein are recognized.

A positive leukocyte test should always be an indication for microscopic follow-up. Even in case of a negative follow-up the diagnosis of pathological leukocyturia remains highly probable, especially when low specific gravity and/or high $\mathrm{pH}$ clearly suggest lysis of the leukocytes.

6. Brühl, P., Mikolai, D. \& Vahlensieck, W. (1979) Urologe A $18,278-283$.

7. Keller, H. \& Koller, P. U. (1982) Med. Lab. 35, 67-72.

8. Kutter, D. (1980) Dtsch. Med. Wochenschr. 105, 12461249.

9. Schaller, G. (1983) Ärztl. Lab. 29, 83-89.

10. Stansfeld, J. M. \& Webb, J. K. G. (1962) Arch. Dis. Child. $37,257-260$.

11. Kutter, D. (1980) Ann. Biol. Clin. 38, 179-182.

12. Kutter, D., Klemmer, L. \& Risch-Thoma, S. (1982) Ärztl. Lab. 28, 17-20.

13. Kutter, D., Figueiredo, G. \& Klemmer, L. (1986) Bull. Soc. Lux. Biol. Clin. 7, 92-105.

Prof. Dr. D. Kutter

Postfach 748

L-2017 Luxemburg 